\title{
USING BLOCKCHAIN TECHNOLOGY IN HUMAN FOOD CHAIN PROVENANCE
}

\author{
MIAO GUO, XIAO JIN LIU \& WEI ZHANG \\ GBS Asset Acceleration, IBM China Development Labs, China
}

\begin{abstract}
The traditional way of tracking food quality through the supply chain can be a difficult task. The most essential issue is food contamination. It often takes weeks even months to identify when and where a food product was contaminated. Another critical point is complex overlapping distribution and processing ecosystem involving farms, distributors, retailers and consumers, which makes it difficult to assure food provenance. Our SafeFood solution using blockchain and Internet of Things technologies can quickly track a food product's process from farm to storage shelf. In the context of food supply, a blockchain could be used to keep track and history of every single item processed throughout the supply chain. With the Hyperledger Fabric, all the food information is collected into the blockchain throughout every step in the supply chain to offer a more efficient way to figure out when and where food items were contaminated, which can help producers the limited contagions by public health officials . The benefits of more transparent food supply chain include: (1) increase the trust multiplied by each participant in food supply chain; (2) identify the source of compromised food and reduce the unnecessarily broad recall, (3) improve the co-ordination in food supply chain; (4) increase operation efficiency; (5) provide better food safety, more freshness and avoid food fraud for customer.
\end{abstract}

Keywords: blockchain, Hyperledger Fabric, Internet of Things, food provenance.

\section{INTRODUCTION}

The traceability of food from 'farm to fork' is a complex distribution and processing ecosystem involving farms, distributors, retailers and consumers, which makes it difficult to assure food provenance. In order to address this issue, the SafeFood solution uses the advantages of blockchain and IoT technologies to keep track and history of food item processed throughout the supply chain and accessible by each party in supply chain to record food processing steps.

Take the case of beef cattle. If the growing states and shipments of beef cattle are recorded in a blockchain, at least in theory, the blockchain record would make it easier to find out where the beef cattle was farmed, and which shipments were exposed and delivered. The blockchain technology could also help companies and public health officials determine which communities might have been exposed.

This paper describes one business scenario of food supply chain provenance and explains how to use blockchain and Internet of Things (IoT) technologies together to build up this end-to-end solution, in order to make sure the solution can be achieved and be reused in any other similar business scenario.

\section{SOLUTION BRIEFING}

2.1 Key technology briefing

\subsubsection{Blockchain}

- Blockchain technology can be used to share a ledger across the business network. The network will be closed (accessible only by the parties concerned), permissioned 
(so only authorized participants can join), private (using cryptographic technology to ensure that participants only see what they are allowed to) [1].

- The shared ledger will be stored in a safer and more robust way, since it is replicated and distributed. There will be consensus across the network, where the provenance of information is clear and transparent. Transactions will be immutable (unchangeable) and final.

- This technology has the potentials to save time, lower cost, reduce risk and increase trust for organizations which share transactional or ledger information across an ecosystem. The relevant companies or parties can therefore operate or participate within an ecosystem, marketplace, network or linked ecosystems.

- Compared with the traditional method that records the traceability information into database, the solution using the Blockchain technology can better achieve tamperproofing with encryption technology.

\subsubsection{IoT}

- IoT is a network of interconnected devices that are embedded with unique identifiers, such as electronics, software, sensors and actuators, etc. The interconnected devices are also embedded with network connectivity so that they can collect and exchange data. Using the IoT technology, the connected objects can be sensed and controlled remotely. When the interconnected devices become responsive, the efficiency, accuracy, and economic benefit will be improved.

\subsubsection{Combination of IoT with blockchain}

- With Blockchain and IoT, a platform will have greater transparency of true history and greater trust since no single party owns the whole historical data. Besides, information can be shared more efficiently [2].

\subsubsection{Hyperledger Fabric}

- Hyperledger Fabric is a blockchain framework and one of the Hyperledger projects supported by The Linux Foundation. Hyperledger is an open source collaborative effort created to advance blockchain technology by addressing important features for a cross-industry open standard for distributed ledgers. Our SafeFood solution uses Hyperledger Fabric V0.6 as our blockchain implementation framework.

\subsection{Solution briefing}

- In SafeFood solution, five participants are involved, i.e. Farmer, Processor, Retailer, Consumer and Regulator. This solution helps build up a platform where information is shared and business rules are executed automatically.

- Farmer: Farm is the place where beef cattle is farmed and the farmers are responsible for recording the new-born beef cattle; monitoring the living status by IoT technology; recording the vaccination status; recording the beef cattle's basic information like weight when beef cattle is fully grown up; and recording the transfer information like processor name, transfer date and time when the beef cattle is transferred to processor.

- Processor: Processing factory is a place where the beef cattle were processed to beef. The processor must record the processing information, including when, how and who; record the meat quarantine information; and record the detail information in transferring the beef to retailer, like transferred to which retailer and when, etc. 
- Retailer: In this stage, the beef meat is sliced and packaged, and this process need be recorded in Blockchain. Inputting the slices information helps generate the QR code, which can be printed and attached on the package. The staff of the retailer, responsible for attaching the right $\mathrm{QR}$ code to the beef meat package. When the beef slice was sold, retailer need record the selling information, including date and time, etc.

- Consumer: Consumers will be able to query the supply chain of the beef meat via scanning the QR code on the meat package by a mobile app. The web UI is another way to query the supply chain information.

- Regulator: It can be government or a third party of food safety provenance organization. The solution can give hints of which participants should be paid more intention or which deal between the participants should be checked.

\subsection{Solution demonstration}

For consumers, after they bought the beef from supermarket, they can easily get the beef supply chain information by scanning a QR code printed on the packaging bag. In China, QR code is very common in daily life. People can pay bills or share information via scanning QR code. We adopt this technique as the interaction channel with consumers.

\section{SOLUTION DEVELOPMENT}

\subsection{Architecture}

The architecture of SafeFood solution is a hierarchical structure which has 8 different components, where Web Portal/Mobile App acts as user access channel, Device and IoT

\section{Regulator}

Query Supply Chain Via Web Page

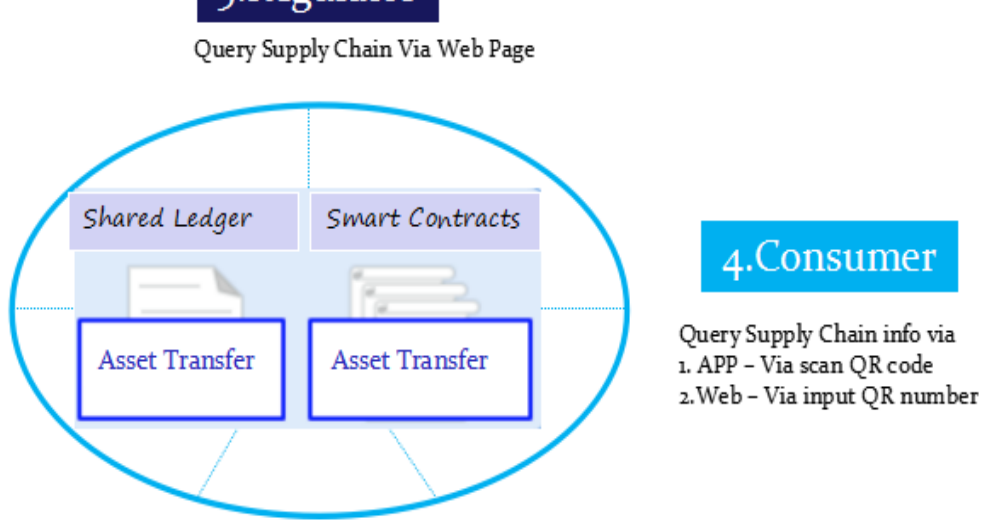

\section{Consumer}

1.New Beef

2.Beef Live Status(IOT)

3.Beef Feeding Record

4.Beef Vaccination

5.Beef Full Grown Record

6.Beef Transfer

\section{Processor}

1. Processing(Slaughter)

2. Quarantine Beef

3. Beef Transfer

\section{Retailer}

1. Beef slice and Packaged with $Q R$ code

2. Beef slices sold out

Figure 1: Major participants of safe food business alliance. 


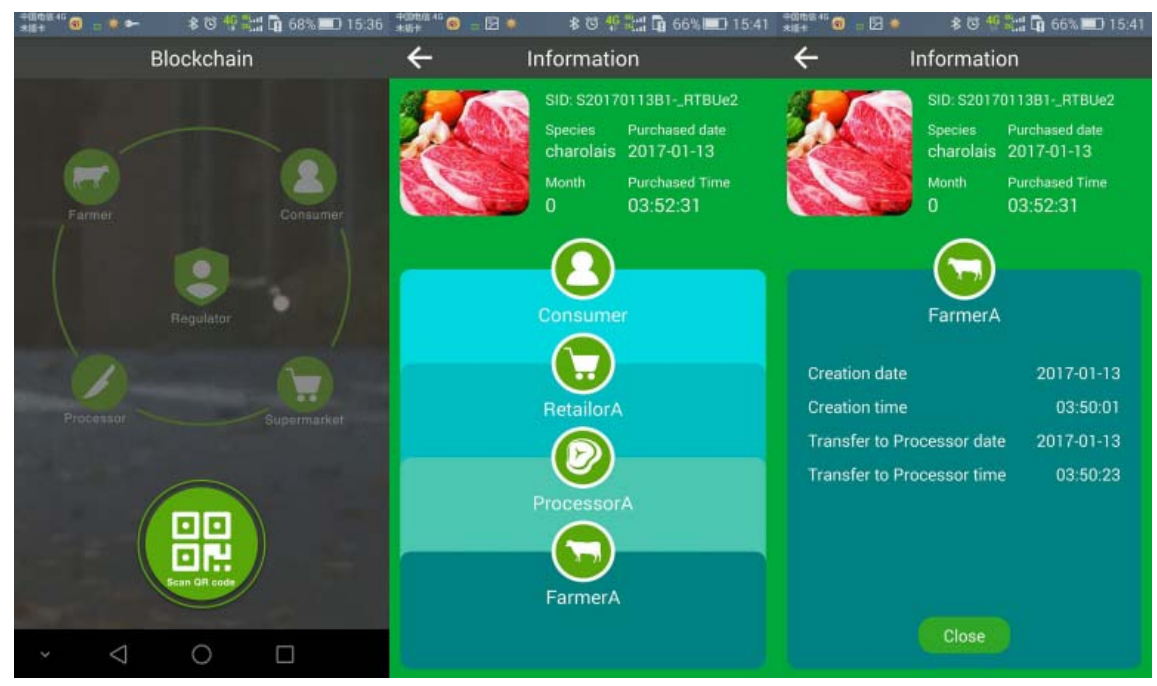

Figure 2: Mobile app.

Platform and Backend Services provide the implementation of business logic. Smart Contract (Chaincode) is the core component of Blockchain. The Data Storage stores both real-time NoSQL data and traditional data. Another two components are Infrastructure and Operating System [3].

- Business application: Web and mobile app enables users to execute the business process. This is a highly user-centred design and more than one app can be used with a smart contract.

- IoT platform: It can connect and manage the devices and receive and send the message. It generally also has the functions of data analytics and risk management. Besides, it also provides the data for the other services. Typically, IBM has a Watson IoT platform that has all the above functions.

- Device: It means IoT device here, such as sensor, phone and network camera, which can be connected to the internet and send/receive the messages. They can get such information as temperature, humidity, videos/pictures taking and sending.

- Backend services: This provides backend services implemented by node.js, including user management, authentication, device connection, management, data analysis and processing.

- Smart contract (Chaincode): This encodes the business process into the transaction that creates blocks on the chain. This is the underlying Fabric that realizes the attributes of consensus, immutability, provenance and finality.

- Data storage: RDBMS for Personally Identifiable Information (PII), or Sensitive Personal Information (SPI) and NoSQL DB and/or file system for processing realtime data captured by IoT devices, including video/image data.

- Operating system: The system on which the Fabric runs, e.g. Linux.

- Infrastructure: The hardware used to host the software, e.g. IBM Cloud. 
According to the architecture diagram (Fig. 3), the major process flow of SafeFood solution includes:

- The devices are registered on IoT Platform, then real-time messages are sent to IoT platform and responsible for collecting beef cattle's living status.

- Backend services receives the data from IoT platform then dispatches data to different processing components, e.g. storing videos/images in file system for further image analysis, storing real-time raw data in NoSQL database as the historical data, and storing other transaction data in traditional database for blockchain process.

- Backend services routes the key data to blockchain ledger.

- Smart contract will be triggered to put the data in the ledger of blockchain or get the data from the ledger.

- Bulk files data should be mapped with the data in blockchain. The disadvantage of storing data by database is that it is easy for someone to do evil things when he has the admin rights of database, or some internet hackers intrudes the database to tamper the data. By contrast, blockchain system with encryption and consensus is very safe, but it is not convenient to save the bulk files, such as videos, images, or large-size text file. In this way, bulk files also can be saved and also verified by the hash code which is saved in blockchain.

- User can use backend services to send the request to register and identity them with Fabric CA, which is a Certificate Authority (CA) for Hyperledger Fabric.

- Web portal and Mobile app can invoke the Backend services to get the data that they want, and they can get the data.

All the above components should be deployed in a cloud, like IBM Cloud, or the physical machine with basic infrastructure, like storage and network, etc. Consensus algorithm runs and compares results across the peers in blockchain network. If the peers agree based on the policy, the changes to the world-state database will be committed, and the data will be saved to ledger.

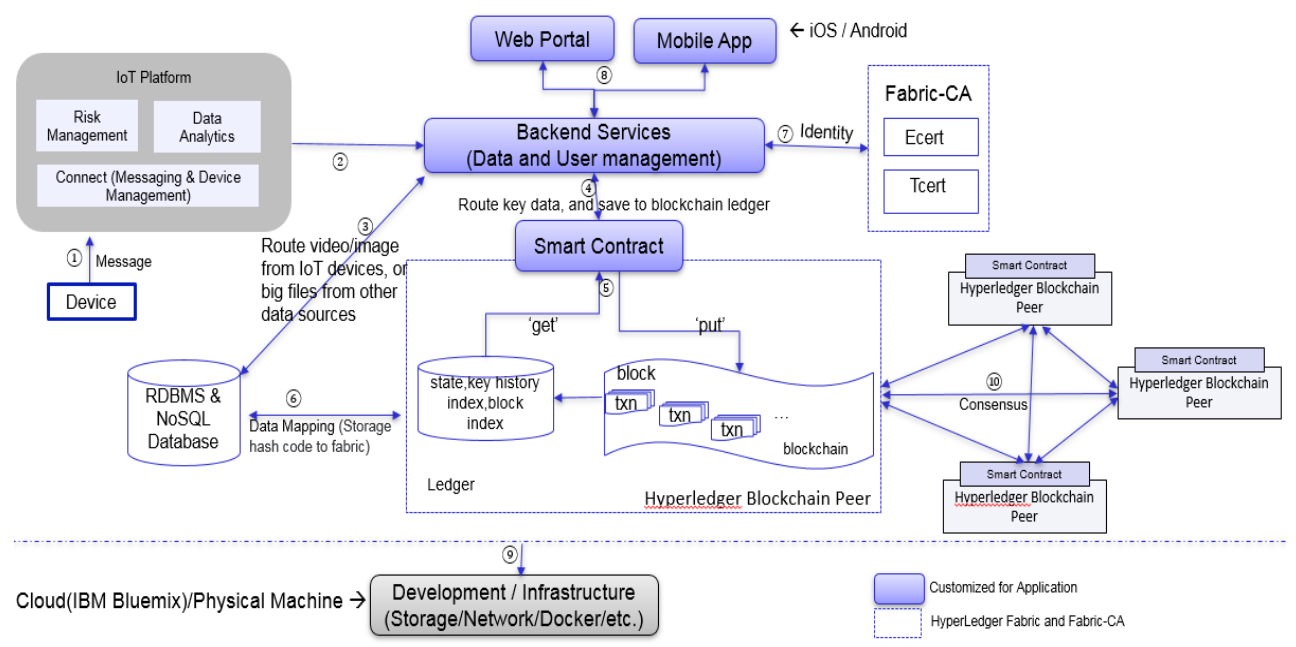

Figure 3: Architecture diagram. 


\section{CONCLUSIONS AND DISCUSSIONS}

This paper introduces a food pinpoint source solution based on Blockchain and IoT technologies. The technologies and methods presented in this paper are commonly used in practice, and worthy for the development of other similar systems.

In this solution, the adopted product is beef cattle. According to the requirements, the product can be rice, tea and chicken, etc., where the basic functionalities in this solution can also be applied. At the same time, some new features need to be implemented, e.g. if the product is rice, the packaging feature should be added to farmer's component. At the same time, the business process may need to modify according the actual situation.

During the tracking process, we use the IoT technology to ensure the products are not changed. Farmer needs to record the product's living status, which might also be monitored by the potential buyer. So, the best way is to use highly sensitive sensor to track animal behaviours, and during transferring process, the bar code or QR code will be used to help identify the product. On the other hand, authorities should be involved in the eco-system and business process to give authoritative certification after random check or routine check.

In China, the government has food safety laws and the laws on the quality and safety of agricultural products. Regulators will take quality inspection for food and agricultural products by sampling inspection. The result of quality inspection can be stored in our solution. Then our SafeFood solution not only has the capability to resolve the problem of sharing information between the upstream and downstream of the supply chain and among consumers, but also has the capability to give more confidence to consumers. It's a more efficient way to share information. The transfer time can be reduced. And consumers can have more refresh food.

\section{REFERENCES}

[1] What is Blockchain? Understand the fundamental of IBM Blockchain. Online. www.ibm.com/blockchain/what-is-blockchain.html

[2] Implement IoT and blockchain for accountability and security. Online. www.ibm.com/ internet-of-things/spotlight/blockchain

[3] Hyperledger Fabric Architecture. Online. https://w3-03.ibm.com/services/lighthouse/ documents 